\title{
Electrical and topological drivers of the cascading failure dynamics in power transmission networks
}

\author{
Alberto Azzolin a, Leonardo Dueñas-Osorio b, *, Francesco Cadini ${ }^{\mathrm{d}}$, Enrico Zio ${ }^{\text {a, c }}$ \\ ${ }^{a}$ Dipartimento di Energia - Politecnico di Milano, Piazza Leonardo Milano, Italy \\ ${ }^{\mathrm{b}}$ Department of Civil and Environmental Engineering, Rice University, 6100 Main Street, Houston TX 77005, United States \\ ' Chair System Science and the Energy Challenge, Fondation Electricite' de France (EDF), Centrale Supélec, Université Paris-Saclay, Grande Voie des Vignes, 92290 Chatenay-Malabry, France \\ d Dipartimento di Meccanica - Politecnico di Milano via La Masa 1 - 20156 Milano, Italy
}

\begin{abstract}
To systematically study key factors affecting cascading failures in power systems, this paper advances algorithms for generating synthetic power grids with realistic topological and electrical features, while computationally quantifying how such factors influence system performance probabilistically. Key parameters affecting line out- ages and power losses during cascading failures include line redundancy, load/generator layout and re-dispatch strategies. Our study combines a synthetic power grid generator with a direct current (DC) cascading failure simulator. The impact of each of the factors and their interactions unravel useful insights for interventions aimed at reducing the probabilities of large blackouts on existing and future power systems. Moreover, conclusions drawn from a spectrum of different power grid topologies and electrical configurations offer more generality than typically attained when studying specific test cases. Line redundancy and distributed generation appear as the most

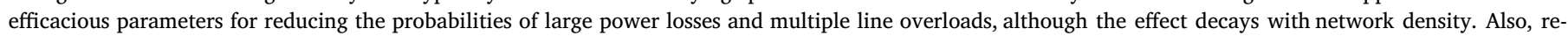
dispatch strategies are critical on the distribution of the cascading failure size in terms of line failures. These and related results provide the basis for probabilistic analyses and future design of evolving power transmission systems under uncertainty.
\end{abstract}

\section{Introduction}

The impact that a power transmission system's topology and associated electrical features have on overall system reliability is still not well understood, especially when their joint effect is considered during cascading failure events. And even when considering a fixed network topology, many different electrical configurations and states are possible, as placements of generation units or dispatch strategies result in different power system dynamics and associated reliability considerations.

Power system reliability research encompases the study of cascading failures, since these failure dynamics have proven serious in economic and social terms [1]. Standard practice in the study of cascading failures is to apply power or network flow models to a limited set of test systems to understand evolving system behavior [2-7]. Also, there are topological studies for simplified yet analytical explorations of system properties, typically based on samples of randomly generated networks when the electrical data are not available [8-11]. However, the probabilistic analysis of power systems subjected to contingencies is still limited, of- ten without the joint effects of topological and electrical configurations (e.g., layout, element siting, re-dispatch). Thus, conclusions drawn to date under probabilistic models, while valuable, remain not easily generalizable. Our work attempts to bridge this gap by applying a cascading failure model based on an $N$-2 contingency analysis with Direct Current (DC) power flow analyses on a sample of synthetic yet realistic power networks generated to capture some of the topological as well as electrical and probabilistic features of power systems [12].

While relying on DC power flow adds realism to the cascading failure model, it also requires that the synthetic networks be adequate electrically. Hence, we extend the algorithm by Wang et al. [12] to produce synthetic power grids suitable for DC power flow computations. The number of features characterizing power grids is high, making the impact analysis of all of them prohibitive. Consequently, we group key features of power grids into three different macro-areas based on known sensitivity analyses $[2,13]$ : the underlying topology, the electrical properties of its components, and the control rules governing the system. This grouping is also consistent with the way each synthetic power grid is generated in our study, as the topology is constructed first, then the

\footnotetext{
* Corresponding author.

E-mail address: leonardo.duenas-osorio@rice.edu (L. Dueñas-Osorio).
} 
topology is enriched with the electrical parameters (e.g., impedances, power demand and supply levels, generation siting, etc.), and finally, the dispatch/control rules are embedded into the cascading failure simulator.

At the topological level, the line redundancy of the network is chosen as input variable, since adding lines is the baseline approach for decreasing systems' congestion and has a straightforward interpretation from an infrastructural point of view. At the electrical level, the generator/load buses layout is considered a key input that we vary in order to compare various degrees of centralization, from clustered to a more dis- tributed siting of loads and generators. The interest in power generation layout lies in the evolution towards smart grids $[1,14]$. Finally, from the operational point of view, we consider two different power re-dispatch strategies.

We carry out computational experiments from full factorial designs to study the effect of key input parameters and offer general insights [15]. The input variables involved are fully crossed with each other, allowing us to gather data on cascading failure effects. Since this approach relies on a catalogue of power grids instead of a particular one, the conclusions drawn have more general appeal.

As for system performance, we study the reliability of the power grid, understood here as the ability of the network to deliver power to customers (via a power loss metric), while the physical infrastructure integrity is preserved (via counts of outages across lines) [1]. The computational experiments that follow, allow us studying power loss and line outages probabilistically, particularly as the topological, electrical and control parameters of the synthetic networks vary. This work thus complements two of the approaches that dominate existing literature: one general and often probabilistic, but limited to topological analyses $[16,17]$, and the other comprehensive in its electrical aspects but specific to a system model $[18,19]$. We aim at a middle point, where we use DC power flows and also study a variety of system configurations and dispatch strategies, so as to gain generalizable insights for future operation management and reliability-based design of evolving power networks.

The rest of the article is organized as follows: Section 2 describes our global strategy to study evolving infrastructure, particularly via computational experiments. Section 3 describes the power network generation procedure and our updates to make key system parameters tunable. Then, Section 4 describes our cascading failure model. Section 5 provides simulation results, and Section 6 discusses outputs and draws insights for network operation. Section 7 concludes the paper and provides ideas for future research.

\section{Global strategy for studying evolving power systems}

To explore how key inputs affect cascading failures in power systems, we perform a full factorial experimental design to elicit general conclusions. Appropriate variables and models for our experimental designs as discussed next.

\subsection{Full factorial design}

Throughout our work, we will deal with a power grid object defined as follows:

$\mathcal{N}=(V, E, Z, P D, P G, P M A X, C)$

where $V$ is the set of buses, $E$ is the set of lines, and $G=(V, E)$ is the power grid topology. Vector $\boldsymbol{Z}$ captures the impedances of the lines, while $\boldsymbol{P D}, \boldsymbol{P G}, \boldsymbol{P M A X}$ are the power demand, supply, and maximum supply of the nodes in $V$, respectively. Vector $C$ denotes the capacity of the lines. We define the subset $\{\boldsymbol{Z}, \boldsymbol{P D}, \boldsymbol{P G}, \boldsymbol{P M A X}\}$ as the electrical properties of the power grid.

Our goal is to determine which parameters affecting $\mathcal{N}$ in Eq. (1) are best at reducing cascading failures, so as to translate findings into design
Table 1

Planning matrix of the full factorial experiment with each row representing a different factor and each column a different level.

\begin{tabular}{llll}
\hline Factors/Levels & 0 & 1 & 2 \\
\hline Line redundancy & $\zeta=-1$ & $\zeta=0$ & $\zeta=1$ \\
Generators layout & $K=0$ & $K=0.5$ & $K=1$ \\
Re-dispatch & $R:=$ Proportional & $R:=O P A$ & \\
\hline
\end{tabular}

guidelines generalizable to evolving power grids. In particular, we consider factors such as: line redundancy $\zeta$, the loads/generators layout $K$, and re-dispatch strategies. Note that topology and electrical features are a function of these factors, such that $E(\zeta), \boldsymbol{P D}(K), \boldsymbol{P G}(K)$, and $\boldsymbol{P M A X}(K)$.

In particular, $\zeta$ will vary in the range $[-1,1]$ interpolating electric networks generated by the RT-nested Small World (RT-nestedSW) algorithm [12], where a realistic topology corresponds to $\zeta=0$, an associated spanning tree to,$\zeta=-1$, and a greedy triangulation (or dense planar network) to $\zeta=1$. In this way, we are able to produce power grid structures with varying levels of line redundancy. Parameter $K$ will vary in $[0,1]$, and is used to produce power grids with increased decentralized generator layout as $K$ increases.

In addition, we analyze the impact on cascading failures of different re-dispatch strategies. The term re-dispatch refers to the action of changing the power supply or demand at the power grid's nodes in real time, so that the total supply and demand are balanced. We compare two re-dispatch strategies in our experiments. First, a proportional strategy where power supply and demand at the nodes changes proportionally to their initial values, and deemed as a baseline strategy. Second, we consider the OPA model [20], which is a simplification of how an operator might intervene in a realistic system when facing complex contingencies. It consists of an optimization routine which minimizes the power losses, subject to constraints in power carrying limits. The OPA model has been used in applications with fast dynamics and long term evolution of power transmission systems [4].

The computer experiments are set as follows: power grids with different values of $\zeta$ and $K$ are generated with our extended RT-nestedSW algorithm, for all their possible combinations. For each of these power grids, two separate $\mathrm{N}-2$ contingency analyses are performed, employing one of the two re-dispatch strategies each. This procedure is tantamount to a balanced full factorial experimental design [15], which unravels the single and joint effects of input factors. Table 1 shows the planning matrix of the experiment.

2.2. Response variables

We compute the total power not served $P_{\text {loss }}^{\left(l_{i}, l_{j}\right)}$ and the number of line failures from cascades $N_{\text {fail }}^{\left(l_{i}, l_{j}\right)}$, following $N$-2 contingency analyses on power grids in $\mathcal{N}$, as associated to a pair of failed lines $\left(l_{i}, l_{j}\right)$. Hence, $\mathcal{N}$ evolves from its initial equilibrium state $\mathcal{N}^{0}$ into a new state $\mathcal{N}^{\left(l_{i}, l_{j}\right)}$ following the dynamics we model trough subsequent Algorithm 4.1. This procedure is then repeated with replacement for all distinct pairs of lines belonging to $\mathcal{N}$. We then define the total $N-2$ contingency analysis effects as power loss $P_{\text {tot }}$ and line failures $N_{\text {tot }}$ as follows:

$$
\begin{aligned}
& P_{\text {tot }}(\mathcal{N})=\sum_{\left(l_{i}, l_{j}\right) \in \mathcal{N}} P_{\text {loss }}^{\left(l_{i}, l_{j}\right)} \\
& N_{\text {tot }}(\mathcal{N})=\sum_{\left(l_{i}, l_{j}\right) \in \mathcal{N}} N_{\text {fail }}^{\left(l_{i}, l_{j}\right)} .
\end{aligned}
$$

Note that $P_{\text {tot }}$ and $N_{\text {tot }}$ are computed for each power grid topological and electrical configuration as well as re-dispatch strategy. Knowing how these performance indicators vary in correspondence to different 
configurations of input factors allows us to understand their global impact on cascading failure vulnerability and associated probabilities of occurrence.

\section{Synthetic power grids for computational experiments}

The following subsections review the procedure to generate new network topologies in agreement with real power grids. Then, the authors explain the role of line redundancy and generator layout factors $(\zeta, K)$, which enable sensitivity analyses.

\subsection{Topological properties of power networks}

Consider a power grid topology $G=(V, E)$, with $|V|=N$ as the number of generation sources, aggregate loads at the substation level and transmission nodes, and $|E|=m$ as the number of links or transmission lines. Research has shown that commonly used synthetic graph structures such as small world [21], scale free [22] and random networks are not able to capture the topological features of real power grids $[12,17,23]$. For example, Wang et al. [12] found that power grids have different connectivity scaling laws than standard small world graphs. In fact, the average nodal degree $\langle k\rangle$ is constant and does not scale with the network dimension, as would be the case for the small world model [21]. Meanwhile, Cotilla-Sanchez et al. [23] noticed that the average path length $\langle l\rangle$ scaling properties of real power grids are in between regular grids and small world networks. Overall, these and other studies call for models that capture system topology as well as functionality consistent with practical power systems $[12,16,17]$.

\subsection{The RT-nested small world model}

Wang et al. [12] postulated nesting several small-world (SW) subnetworks into a regular lattice to retain electrical system features while using simple topologies that exhibit realistic scaling properties $[16,17]$. Another power network generator was used in Purchala et al. [24] to test DC power flow accuracy in active power considerations. They found bounds for the lines reactances and resistance ratios, below which the DC power estimation has unacceptable high errors. The RT-nestedSW model has also been refined by $\mathrm{Hu}$ et al. and Genger et al. $[25,26]$ in order to produce synthetic DC and AC power flow test cases. While synthetic models continue development, we adopt the basic RT-nestedSW perspective and expand as needed to realize our experimental design. The method [12], and associated extensions are summarized in Table 2.

The input parameters necessary to initialize the algorithm in Step $1\left\{d_{0},\langle k\rangle, N\right.$, and the number of subnetworks $\}$, are estimated from the IEEE 118-node system [27] as our reference realistic network throughout the study. Hence, Steps 1-4 generate power networks $G=(V, E)$ with topological properties consistent with real systems. Then, in Step 5 , line reactances are randomly generated from a specified heavy-tailed distribution fitted to the real power grid that one wishes to use as a reference. In this work, a Gamma distribution is found to fit well the impedances of the IEEE 118-node system [12]. For each link of the network we sample an impedance value. Then, the sampled values are sorted by magnitude in ascending order and grouped into: local links, rewire links, and lattice connection links according to corresponding proportions. Line reactances in each group are then assigned randomly to the corresponding group of links in the topology. We will denote with $Z^{G}$ the reactances vector associated to a particular topology $G$, where the component $Z_{l}^{G}$ denotes the reactance of line $l \in E$.

With the previous Step and Step 7, we extend the RT-nestedSW algorithm and assign electrical properties, $P D, P G, P M A X, C$-necessary for DC power flow computations and cascading failure analysis as described later. Networks as described constitute the baseline systems corresponding to a line redundancy level of $\zeta=0$ (Table 1 ). Note that changes in $\zeta$ result in changes to the parameters $d_{0},\langle k\rangle$ of Step 1 . We explain next
Table 2

Summary of the RT-nested Small World model (RT-nestedSW) and extensions.

\begin{tabular}{|c|c|}
\hline Pseudo-Code & RT-nested small world model generation \\
\hline Step 1 & $\begin{array}{l}\text { Select the desired number of subnetworks, their } \\
\text { properties, and a geographical distance } \\
\text { threshold } d_{0} \text {. }\end{array}$ \\
\hline Step 2 & $\begin{array}{l}\text { Construct subnetworks as follows: for each node } \\
i \text { select links at random (their number follows } \\
\text { a Poisson distribution with mean }\langle k\rangle \text { ) from } \\
\text { nodes } j \text { belonging to } N_{d 0}^{(i)}=\left\{j: d(|j-i|)<d_{0}\right\} \\
(i, j \text { are the indices of the nodes considered, } \\
\text { and } d(\bullet) \text { is a distance operator). }\end{array}$ \\
\hline Step 3 & $\begin{array}{l}\text { Rewire the subnetwork links using a Markov } \\
\text { chain model. }\end{array}$ \\
\hline Step 4 & $\begin{array}{l}\text { Randomly connect the subnetworks with each } \\
\text { other, through lattice connections. }\end{array}$ \\
\hline Step 5 & $\begin{array}{l}\text { Assign the lines' impedances using a suitable } \\
\text { probability distribution model. }\end{array}$ \\
\hline Step 6 & $\begin{array}{l}\text { Assign power demand and supply to the nodes in } \\
\text { the network. (Extended) }\end{array}$ \\
\hline Step 7 & Assign lines capacities. (Extended) \\
\hline
\end{tabular}

how the line redundancy of these baseline networks is varied to form topologies with levels $\zeta=-1$ and $\zeta=1$.

\subsection{Bounding models}

The range in which baseline topologies are allowed to vary is bounded from below and above by two limiting cases: the power grid's spanning tree (ST) which has a minimum level of lines to guarantee connectivity, and the greedy triangulation (GT) which approximates the maximum number of lines in a two dimensional planar space.

From each baseline network generated with the RT-nestedSW algorithm, $G_{i}=\left(V^{G_{i}}, E^{G_{i}}\right)$ and associated impedance vector $Z^{G_{i}}$, we construct the $S T_{i}=\left(V^{S T_{i}}, E^{S T_{i}}\right)$ and $G T_{i}=\left(V^{G T_{i}}, E^{G T_{i}}\right)$ networks. Note that $V^{S T_{i}}=V^{G_{i}}$, while $E^{S T_{i}}$ is the subset of lines belonging to a spanning tree of $G_{i}$, with their respective impedances. Similarly, $V^{G T_{i}}=V^{G_{i}}$ and $E^{G T_{i}}$ is the superset of lines belonging to the planar triangulation of $G_{i}$, where the impedances of the lines belonging to $E^{G T_{i}}-E^{G_{i}}$ are generated as in Section 3.2.

Factor $\zeta$ varies in the range $[-1,1]$ where 0 indicates the baseline topology $G$ and values in $-1 \leq \zeta \leq 0$ represent the percentage of lines belonging to $E^{G}-E^{S T}$ up to obtaining $G$. Meanwhile, values in $0 \leq \zeta \leq 1$ indicate the percentage of lines belonging to $E^{G T}-E^{G}$ which are added to $G$ up to forming a GT. Fig. 1 shows topologies for $\zeta=-1,0$ and 1 .

\subsection{Siting of loads and generators}

Power demand vector $\boldsymbol{P D}$ and a power supply vector $\boldsymbol{P} \boldsymbol{G}$ across the nodes' set $V \in G$, help determine the layout for generators/loads within the synthetic power grids, whose assignment is controlled by parameter $K$ in Table 1. From the loads perspective, we use as vector of power demand $\boldsymbol{P D}$ a set consistent with the IEEE 118-node system [27]. We only retain the original components of the power supply vector $P \boldsymbol{G}$ and maximum power supply vector $\boldsymbol{P M A X}$ for the 15 major power suppliers, as the IEEE-118 system has generators overrepresented (i.e., several serve as boundary conditions to the larger system from which it was extracted). We complemented available data in terms of PMAX for the IEEE 118 system, with optimal power flow test case data included in Zimmerman et al. [28]. Hence, each component $P G_{i}, P M A X_{i}$, and $P D_{i}, i=1,2 \ldots N$ represents the power supplied, the maximum power that could be supplied, and the power demanded by bus $i$, respectively 


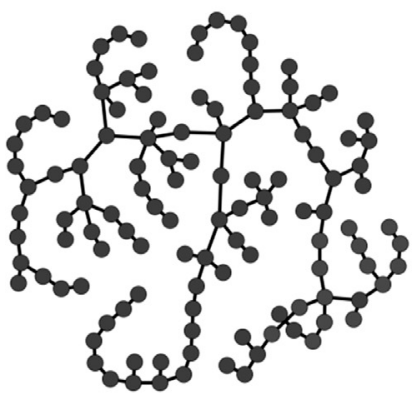

(a)

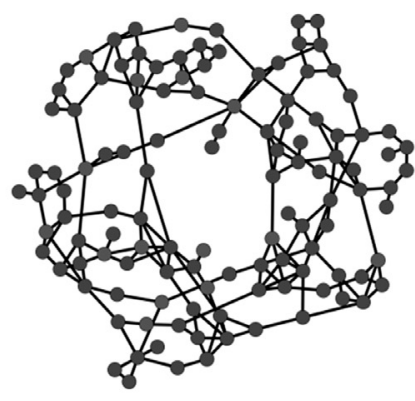

(b)

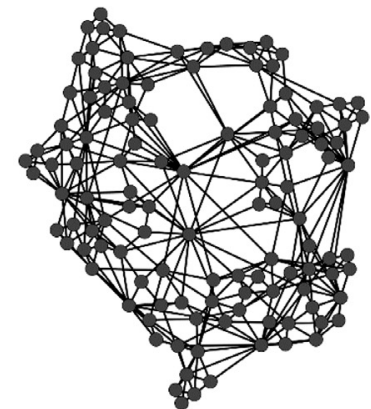

(c)

Fig. 1. Sample topologies with different redundancy levels for the same node set layout: (a) $\zeta=-1(N=118, m=117),(b) \zeta=0$ ( $N=118, m=184)$, and (c) $\zeta=$ $1(N=118, m=369)$.

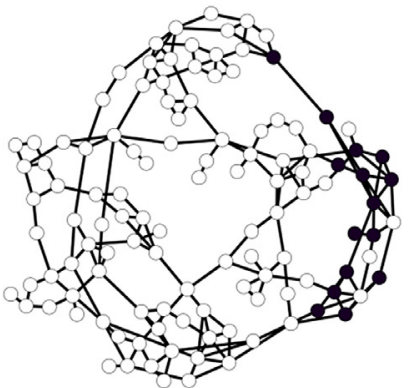

(a)

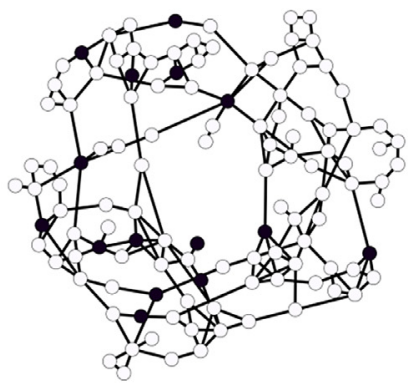

(b)

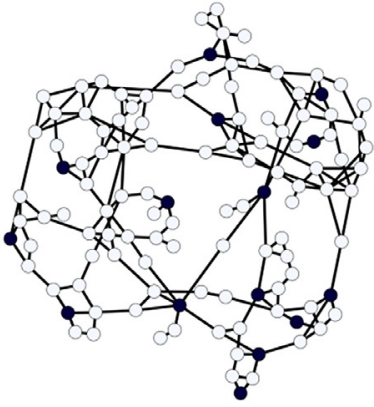

(c)

Fig. 2. Sample networks with different generator set positions according to: (a) $K=0$, (b) $K=0.5$, and (c) $K=1$. Nodes with black fill are generators.

(if $i$ is a load node $P G_{i}=P M A X_{i}=0$ ). Therefore, synthetic power grids have 15 generator buses, 93 load buses and 10 transmission buses, to maintain realistic proportions. We use the following procedure which relies on a spectral clustering algorithm [29] to assign the bus positions of the 15 generators:

1. Select extremes in the power grid as ext $\in V$ (the extremes are the pair of buses most distant from each other), and a threshold distance $d(K)$ (intended as number of links as most lines have similar length).

2. Build the subnetwork $\tilde{\mathcal{N}}=(\tilde{V}, \tilde{E})$ by removing from $\mathcal{N}$ all the buses that are at a distance more than $d$ from ext, and divide it in 15 bus communities $\tilde{V}_{1}, \tilde{V}_{2}, \ldots, \tilde{V}_{15}$ using the spectral clustering algorithm.

3. Select a node at random for each community $\tilde{V}_{i}$, and assign to it a power supply and maximum power supply component.

This procedure forces the generators and the loads to be clustered together depending on:

$d=d_{\min }+K\left(d_{\max }-d_{\min }\right)$

where $d_{\text {min }}$ is the minimum distance (in number of links) where at least 15 buses are at a distance less than $d_{\min }$ from ext, $d_{\max }$ is the diameter of the network and $K \in[0,1]$ is the input factor in our experiment. Note that in this section we are interested in modelling different load/generator geographic layouts. For this reason, Eq. (4) uses the number of links as distance, as the link length distribution is concentrated on a small range of values [30], and thus adequate to capture geographic patterns; alternatives such as electrical distances [31], are also desirable for dynamic analyses, but not necessarily to reproduce geographical layout patterns as in Fig. 2. This figure has three examples of generator positioning for different values of $K$, so as to assist unraveling the impacts of generator siting on cascading failures.

In Eq. (4) as $K$ approaches $0, d$ approaches $d_{\min }$ and only a few nodes close to the network extremes are available to become power generators. This results in most power generators concentrated in a small portion of the power grid, spatially distinct with respect to the rest of the grid. As $K$ approaches 1 more and more nodes become available as generators, hence power can be supplied by buses all over the power grid, and the spatial clustering between generators and loads blurs. In particular, the values of $K$ considered for sensitivity analysis are: $0,0.5$ and 1 (Table 1 ). This factor allow us exploring the effect of spatially distributed versus centralized power generation, which is of interest from a "smart grid" perspective, especially as the mainly centralized power generation structure of existing grids evolves into one that admits decentralization and distributed generation [1]. As the position of power generating units has been found in Pahwa et al. [13] to affect the frequency and voltage stability of power grids, it also determines the paths in DC power flows.

\subsection{Line capacity assignments}

To perform cascading failure simulations, it is necessary to also have transmission lines' capacity data consistent with functional systems. In real power system lines, capacities belong to a set of finite discrete values, while power grids are usually $N-1$ compliant. To produce synthetic power networks with these features, we build a model for the line capacity allocation that differs from a proportional model usually found in the literature $[13,32,5,33]$. Therefore, to sample the capacity $C_{l}$ of line $l$, we base our model on a truncated exponential distribution, Eq. (5), with parameter $\lambda$ estimated from the power flow-to-capacity ratio data of a large real system (we used a Kolmogorov-Smirnov test to confirm that the exponential model was not rejected). Specifically, we studied the Polish grid available in Zimmerman et al. [28] to set our exponential model, as it is one of the most complete power transmission network datasets (note that the IEEE 118 system does not offer such 
Algorithm 4.1

Cascading failure simulator (CFS).

Input: Power network $\mathcal{N}$, initial failures $l_{i}, l_{j} \in E$

1. $\mathcal{N}^{0}=\mathcal{N}$, compute $\boldsymbol{f}^{0}$ vector of power flow in $\mathcal{N}^{0}$

2. $\mathcal{N}^{1}=\left(V, E-\left\{l_{i}, l_{j}\right\}, Z, P D, P G, P M A X, C\right)$

For: $r=1,2 \ldots$ Do

3. Adjust load and generation $R:=\{$ proportional or OPA $\}$ strategy

4. Compute $\boldsymbol{f}^{r}$ power flow vector in $\mathcal{N}^{r}$

5. Identify $O^{r}$ as the set of lines outaged in iteration $r$ If $\left|O^{r}\right| \geq 1$

6. Set $\mathcal{N}^{r+1}=\left(V, E^{r}-O^{r}, \boldsymbol{Z}, \boldsymbol{P} \boldsymbol{D}^{r}, \boldsymbol{P} \boldsymbol{G}^{r}, \boldsymbol{P} \boldsymbol{M} \boldsymbol{A} \boldsymbol{X}, \boldsymbol{C}\right)$

Otherwise: END

details):

$\left(C_{l} \mid f_{l}, f_{l}^{\max }, \zeta\right) \sim \frac{\lambda\left|f_{l}\right| e^{-\frac{\lambda\left|f_{l}\right|}{c}}}{c^{2}\left(e^{\frac{\lambda\left|f_{l}\right|}{C_{\zeta}^{\max }}}-e^{-\frac{\lambda\left|f_{l}\right|}{f_{l}^{\max }}}\right)} I_{\left(f_{l}^{\max }, C_{\zeta}^{\max }\right]^{(c)}}^{(c)}$

where $I$ is the indicator function, $\zeta$ is the redundancy level of the network to which line $l$ belongs to, $C_{\zeta}^{\max }$ is a capacity limit dependent on the line redundancy, $f_{l}$ is the power flowing through $l$ when the DC power flow is computed on the full network, and $f_{l}^{\max }$ is:

$f_{l}^{\max }=\max \left(\left|f_{l}\right|,\left|f_{l}^{1}\right|,\left|f_{l}^{2}\right|, \ldots,\left|f_{l}^{m}\right|\right) \quad l \in E^{\mathcal{N}}$

with $f_{l}^{i}$ as the power flowing through line $l$ when line $i$ is removed from $E^{\mathcal{N}}$, considering $f_{l}^{l}=0$, and $m=\left|E^{\mathcal{N}}\right|$. Note that the support of the probability density function (pdf) in Eq. (5), for different values of $f_{l}$ and $f_{l}^{\max }$, covers capacity values that are finite and guarantee $N-1$ compliance. However, since we want our sample to belong to a discrete set of capacities, we discretize Eq. (5) among equally spaced quantiles: $\left\{\bar{C}_{1}<\bar{C}_{2}<\ldots<\bar{C}_{n^{\zeta}}\right\}$, where $n^{\zeta}$ is dependent on the average number of lines of the networks with redundancy $\zeta$ :

$P\left(C_{l}=\bar{C}_{i} \mid f_{l}, f_{l}^{\max }, \zeta\right)=\frac{e^{-\frac{\lambda\left|f_{l}\right|}{\bar{C}_{i}}}-e^{-\frac{\lambda\left|f_{l}\right|}{\bar{C}_{i-1}}}}{c^{2}\left(e^{\frac{\lambda\left|f_{l}\right|}{C_{\zeta}^{\max }}}-e^{-\frac{\lambda\left|f_{l}\right|}{f_{l}^{\max }}}\right)} I_{\left(f_{l}^{\max }, C_{\zeta}^{\max }\right]}\left(\bar{C}_{i}\right)$.

The distribution in Eq. (7) reflects discrete and finite capacities as seen in real systems. Our line power flow-capacity ratios are distributed in a similar way to the Polish grid as a reference, and are initially $N-1$ compliant. The demanding computation here is for $f_{l}^{\max }$, as one needs to compute the power flow for a network with one of its lines removed, for all of its lines in all the generated networks. After applying consecutively all the procedures just described, a complete set of synthetic DC power grid models $\mathbf{N}$ is obtained whose elements $\mathcal{N} \in \mathbf{N}$ are defined as in Eq. (1).

\section{Cascading failure model}

In the following sections we explain the cascading failure model employed to study all the power grid configurations in $\mathbf{N}$.

\subsection{Algorithm for cascading failure process}

The general steps are illustrated in Algorithm 4.1 (Cascading failure simulator, CFS) based on Bienstock [2]. The algorithm receives as input a power grid object $\mathcal{N}$, and the indices of the initial couple of line failures $\left(l_{i}, l_{j}\right)$ to study $N-2$ reliability compliance. Then, Step 1 computes the power flow at the equilibrium state before initial failures. Step 2 applies the initial failure to the power grid by removing the targeted lines. Then, each iteration $r$ of the For loop corresponds to a failure event in the cascade simulation [i.e., when some lines become overloaded, with $r=1$ corresponding to the initial failure of $\left.\left(l_{i}, l_{j}\right)\right]$. If the initial failure does not lead to a cascade, then CFS 4.1 stops at $r=1$. Step 3 is necessary in order to deal with islanding when line failures break the original network $G$ into multiple connected components which might have an imbalance in power supply or demand [34]. Step 3 is handled by a DC re-dispatch logic that will be explained in Section 4.2. At the end of the simulation, systemic metrics $P_{\text {loss }}^{\left(l_{i}, l_{j}\right)}$ and $N_{\text {fail }}^{\left(l_{i}, l_{j}\right)}$ are computed, and after the algorithm has run for all the couples of lines of the network, $P_{\text {tot }}$ and $N_{\text {tot }}$ are computed as in Eqs (2) and (3).

\subsection{Re-dispatch logic}

During a cascading failure it is possible that an originally connected power network becomes separated in $v$ subnetworks or islands: $I s l_{1}, I s l_{2}, \ldots, I s l_{v}$ [2]. Each island, being a subset of the original power grid $\mathcal{N}$, is defined consistently with Eq. (1) as follows: $I s l_{i}=\left(V^{I s l_{i}}, E^{I s l_{i}}, Z^{I s l_{i}}, \boldsymbol{P} \boldsymbol{D}^{I s l_{i}}, \boldsymbol{P} \boldsymbol{G}^{I s l_{i}}, \boldsymbol{P} \boldsymbol{M} \boldsymbol{A} \boldsymbol{X}^{I s l_{i}}, \boldsymbol{C}^{I s l_{i}}\right) \subset \mathcal{N}$. In principle, islands do not have balanced power supply and demand, thus requiring a readjustment in vectors $\boldsymbol{P G}, \boldsymbol{P D}$ through an operation called re-dispatch. Re-dispatch is not a property of the power grid structure of Eq. (1), and hence it is embedded in Step 3 of the CFS Algorithm 4.1. We could have chosen other control actions; however, re-dispatch is still one of the most common operational actions used to reduce costs and counteract line overloads. Meanwhile, other operations such as transmission switching are mainly applied for planned outage management and costs reduction, but their impact on reliability is only starting to be understood [35]. Hence, at each iteration $r$ of an overloading event, the proportional routine adjusts power in $I s l_{1}, I s l_{2}, \ldots, I s l_{v}$ as follows:

$\forall i \in V^{I s l_{j}}: P G_{i}^{r}=\alpha_{i} P G_{i}^{r-1}+\gamma_{i}, P D_{i}^{r}=\beta_{i} P D_{i}^{r-1}$

where $\alpha_{i}, \beta_{i}$ and $\gamma_{i}$ take different values depending on particular situations:

$\alpha_{i}=\frac{P D_{j}^{r-1}}{P G_{j}^{r-1}}, \gamma_{i}=0, \beta_{i}=1$ if $P G_{I s l_{j}}^{r-1} \geq P D_{I s l_{j}}^{r-1}$

$\alpha_{i}=1, \gamma_{i}=\frac{\left(P M A X_{i}-P G_{i}^{r-1}\right)\left(P D_{I s l_{j}}^{r-1}-P G_{I s l_{j}}^{r-1}\right)}{P M A X_{I s l_{j}}-P G_{I s l_{j}}^{r-1}}$,

$\beta_{i}=1$ if $P M A X_{I s l_{j}} \geq P D_{I s l_{j}}^{r-1} \geq P G_{I s l_{j}}^{r-1}$

$\alpha_{i}=\frac{P M A X_{i}}{P G_{i}^{r-1}}, \gamma_{i}=0 \beta_{i}=\frac{P M A X_{I s l_{j}}^{r-1}}{P D_{I s l_{j}}^{r-1}}$ if $P D_{I s l_{j}}^{r-1} \geq P M A X_{I s l_{j}}$

where $P G_{I s l_{j}}^{r-1}=\sum_{i \in V^{I s l_{j}}} P G_{i}^{r-1}, P D_{I s l_{j}}^{r-1}=\sum_{i \in V^{I s l_{j}}} P D_{i}^{r-1}$ are the total power generated and demanded in island $j$ before the overloading event $r$. Also, $P M A X_{I s l_{j}}=\sum_{i \in V^{I s l_{j}}} P M A X_{i}$ is the total power capacity of the island. This proportional logic is simple by not taking into account the capacities of the surviving lines in the system and is close to a "no-redispatch simulation", particularly as it maintains power balance in the network with limited optimization steps, intervening only in case of islanding. Proportional power generation is still frequently used in practice as in the case of generator disconnections [34]. In the case of fast cascading failures, there could be insufficient time to carry out more sophisticated than proportional interventions. Hence, the proportional logic offers a suitable baseline for comparison with desired optimization based strategies.

In contrast, the OPA model re-dispatches power by solving the following linear program for each island $I s l_{1}, I s l_{2}, \ldots, I s l_{v}$ :

$\forall j \in\{1,2, \ldots, v\}$ solve :

$\min _{\Delta p g, \Delta p d} \sum_{i \in V^{I s l_{j}}} \Delta p g_{i}+100 \sum_{i \in V^{I s l_{j}}} \Delta p d_{i}$

Subject to:

$\forall i \in V^{I s l_{j}}:-P G_{i}^{r-1} \leq \Delta p g_{i} \leq P M A X_{i}$ 


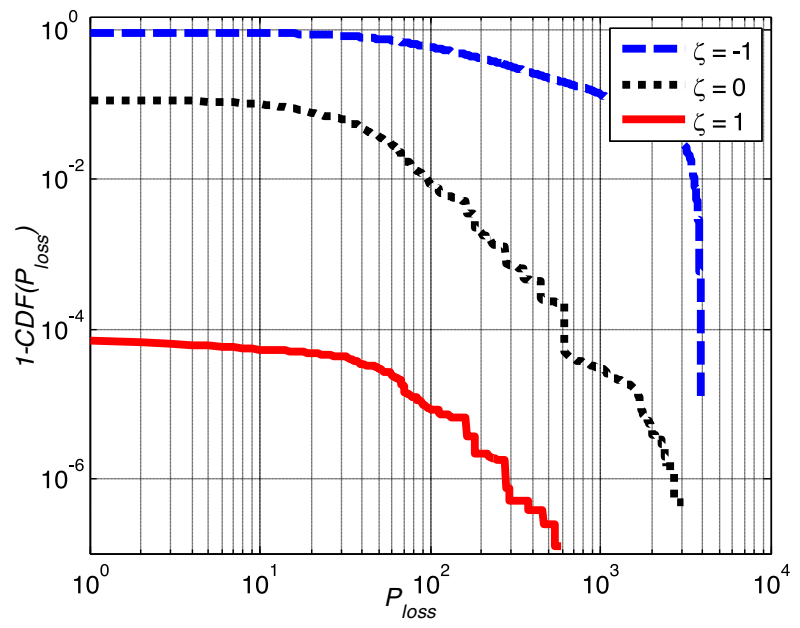

(a)

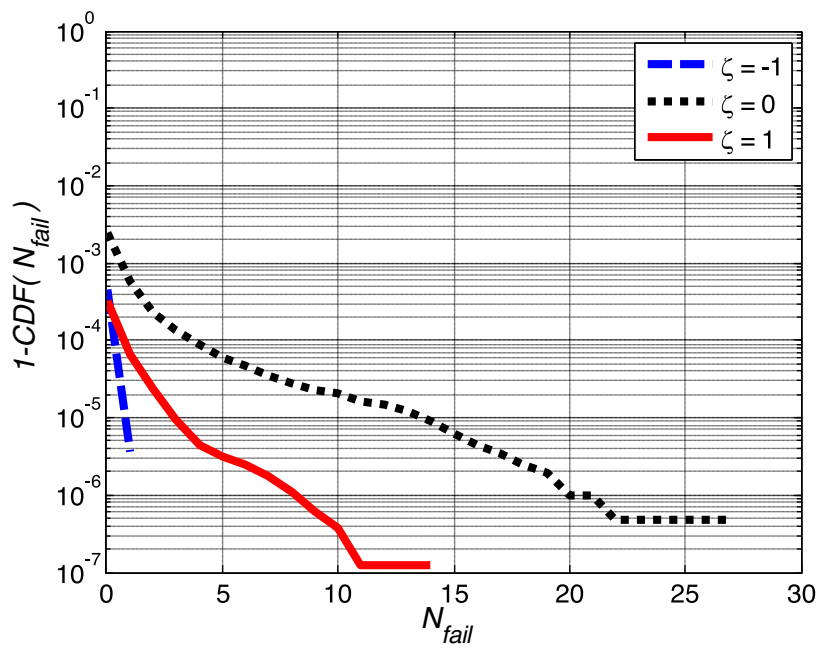

(b)

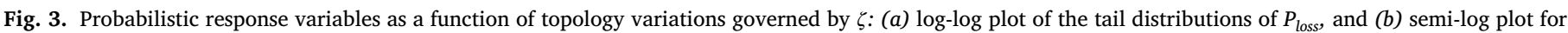
the tail distribution of $N_{\text {fail }}$.

$\forall i \in V^{I s l_{j}}: 0 \leq \Delta p d_{i} \leq P D_{i}^{r-1}$

$\sum_{i \in V^{I s l_{j}}}\left(\Delta p g_{i}-\Delta p d_{i}\right)=\sum_{i \in V^{I s l_{j}}}\left(P G_{i}^{r-1}-P D_{i}^{r-1}\right)$

$\forall l \in E^{I s l_{j}}:-C_{l}-f_{l}+\varepsilon_{l} C_{l} \leq \boldsymbol{B}_{l .}(\Delta p g-\Delta p d) \leq C_{l}-f_{l}+\varepsilon_{l} C_{l}$

where $\boldsymbol{B}_{l}$. is the $l$ th row of the susceptance matrix $\boldsymbol{B}$ of the network. The objective function in Eq. (12) allows to re-dispatch power giving priority to generators by penalizing power demand modifications, as avoided whenever possible in practice. Constraints in Eq. (13) make sure that the power supplied by each generator is always below its maximum capacity, while constraints in Eq. (14) bound the power demanded from each load to its initial value (when the grid is in equilibrium). Constraints in Eq. (15) ensure that in each island, supply and demand are balanced, and constraints in Eq. (16) force the re-dispatch action to respect the surviving lines' capacities. Since in real situations the thermal rating of transmission lines is never known exactly and depends on external conditions as well as intrinsic properties of the material and shape of the conductors, a small error noise $\varepsilon_{l} C_{l}$ is introduced in the nominal capacity value $C_{l} . \varepsilon_{l}$ is randomly sampled from a uniform distribution between -0.01 and 0.01 every time the optimization is performed. After solving the linear program, the power supply/demand of each bus in $I s l_{j}$ is adjusted:

$P D_{i}^{r}=P D_{i}^{r-1}-\Delta p d_{i} \forall i \in V^{I s l_{j}}$

$P G_{i}^{r}=P G_{i}^{r-1}+\Delta p g_{i} \forall i \in V^{I s l_{j}}$

The OPA model is a simplification of how a power grid operator might intervene in re-dispatching power. It has been used to solve optimization problems related to the prevention of blackouts [36], as well as to study the vulnerability of interdependent systems [37].

\section{Computational experiments and results}

\subsection{Impact of topology on cascade failure vulnerability}

Fig. 3 shows the log-log and semi-log plots of the tail distribution of response variables $P_{\text {loss }}$ and $N_{\text {fail }}$ for the original synthetic power grids and the two bounding topologies derived in Section 3.3, representing various line redundancy factors $\zeta$.
As expected, the complementary cumulative distribution function (ccdf) for baseline networks $(\zeta=0)$ is halfway vulnerable between power delivery interruptions in the tree network $(\zeta=-1)$ and the greedy triangulation $(\zeta=1)$ [Fig. 3(a)]. For $\zeta=-1$, the power grids show high probabilities of power losses, as the probability of $P_{\text {loss }}>10^{2} \mathrm{MW}$ is above $50 \%$. Since power losses depend on islanding and bus disconnections in our model, such strategies are at odds when $\zeta=-1$ as only minimal sets of lines necessary to ensure connectivity are present. Hence, each line failure is sufficient to split the grids into multiple connected components and, depending on the position of generators, cause power losses. When $\zeta=0$ and $\zeta=1$, topologies are less sensitive to line failure given more alternative paths to satisfy demands, and in most cases few failures do not alter system-level functional pathways. In fact, the probability of having $P_{\text {loss }}>10^{2} \mathrm{MW}$ after a double contingency is approximately $1 \%$ for $\zeta=0$, and below $0.01 \%$ for $\zeta=1$. However, note that the tail of the ccdf for $\zeta=0$ still reaches values comparable to the worst scenarios in the tree networks, signaling undesirable configurations although at a much lower frequency. No such configuration is present in the greedy triangulation given the short tail. The maximum power losses for the three different network groups are: 3,879 MW for $\zeta=-1,3,098$ MW for $\zeta=0$ and 598 MW for $\zeta=1$ on a total initial power production of 4,377 MW.

The role of line redundancy is different with respect to $N_{\text {tot }}$, where Fig. 3(b) shows that the $\zeta=0$ topologies have higher probabilities of additional line failures after the initial double line contingency. This is because line overloads affect lines belonging to alternative paths, and since in $\zeta=-1$ configurations only one path exists between each couple of buses, no overload due to power flow re-distribution is possible. In contrast, the $\zeta=0$ and $\zeta=1$ topologies can be subject to line failures when the power flow is re-directed. The tail distribution of $N_{\text {fail }}$ is higher for $\zeta=0$ than $\zeta=1$ showing that $N_{\text {fail }}$ is non-linear and non-monotonic with respect to $\zeta$. The $\zeta=1$ networks have a much higher number of alternative paths than the $\zeta=0$ configurations, thus the power flow re-directed by the initial line failures is dispersed among a larger set of possible alternative routes. Specific results show that the maximum number of consecutive overloads for the three different structures is 4 for $\zeta=-1,28$ for $\zeta=0$ and 15 for $\zeta=1$.

\subsection{Impact of generators positions}

Regarding the electrical structure, there is impact from different positions of generator buses via the $K$ factor (spanning from a centralized 


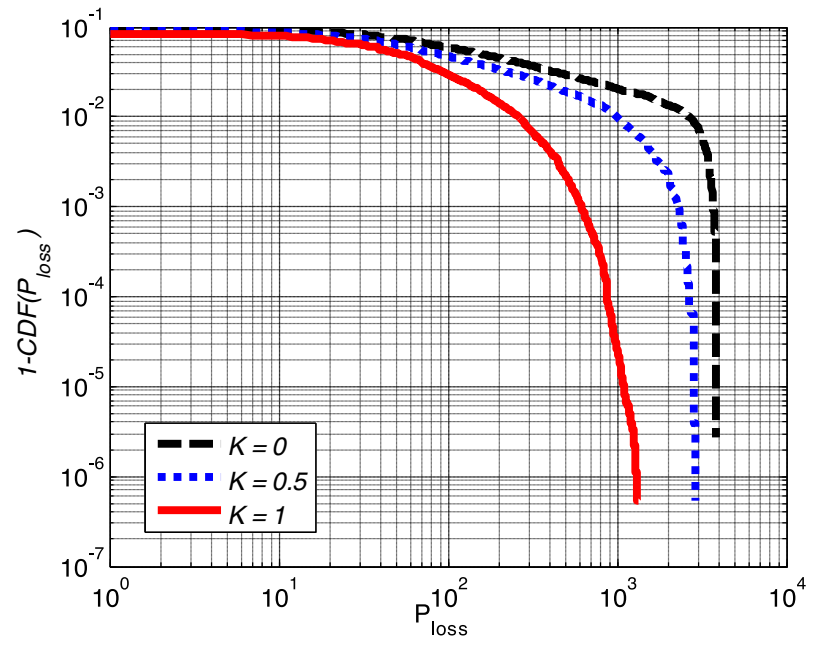

(a)

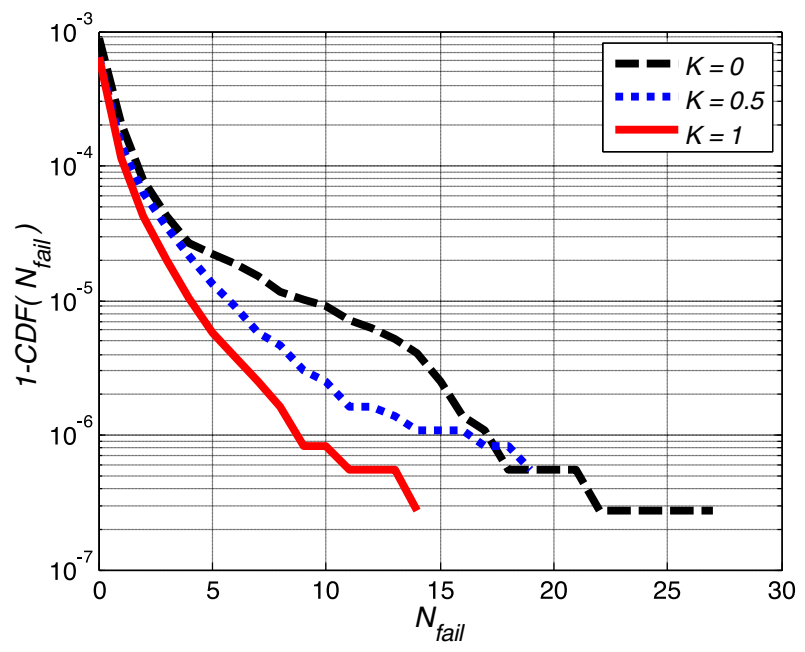

(b)

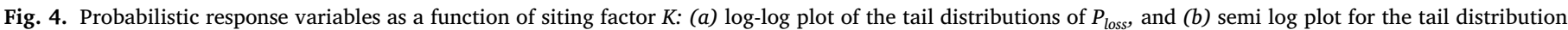
of $N_{\text {tot }}$.

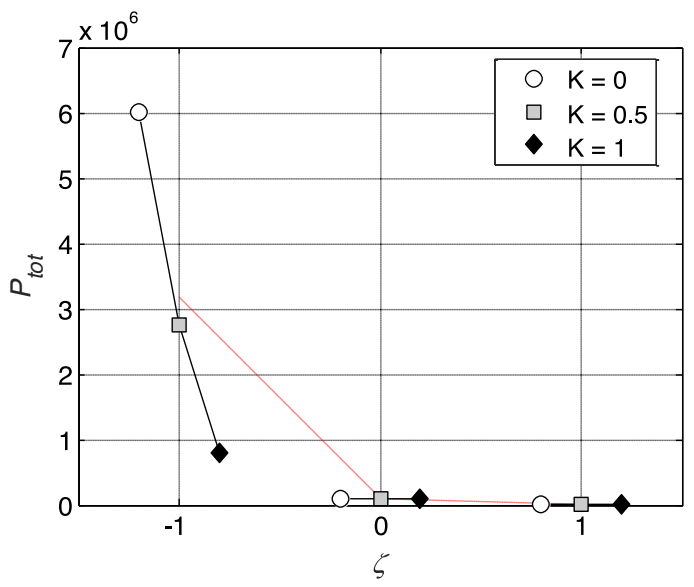

(a)

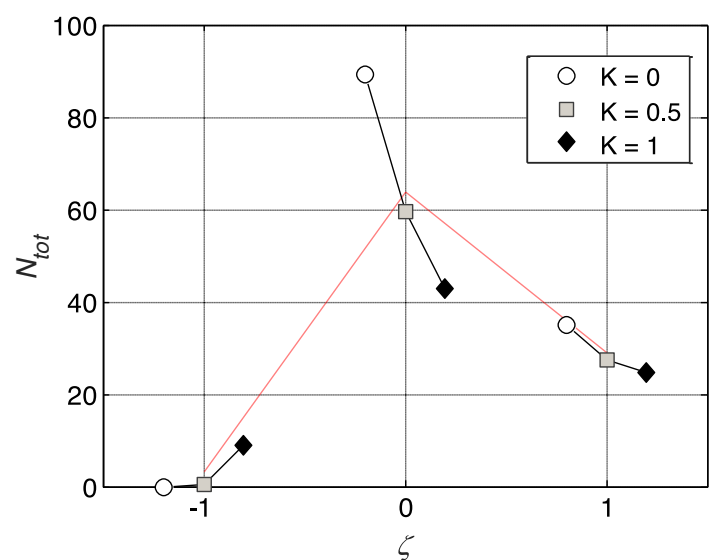

(b)

Fig. 5. Multivariate chart of the group average of (a) $P_{\text {tot }}$, and (b) $N_{\text {tot }}$ with respect to $\zeta$ and $K$.

power supply layout to a distributed one). Fig. 4 shows the log-log plot of the empirical tail distributions for $P_{\text {loss }}$ and semi-log of the same quantity for $N_{\text {fail }}$ for the different levels of factor $K$-here we considered a total of 360 cases.

Fig. 4(a) and (b) show that the probability of both high power losses and additional line failures decreases as $K$ increases (i.e., as we move from a centralized structure to a distributed one). In fact, centralized power generation structures have important tie lines which connect the power supply agglomerates with the power demand sites. Failing these tie lines cause more widespread power interruptions as reflected in the higher values of expected $P_{\text {loss }}$. In contrast, the distributed generation cases $(K=1)$ are more robust since the supply and demand buses are spread through the network and not separable by a few line failures. These general considerations do not apply equally to every topological type, as one can see from the multivariate chart for the $\zeta \times K$ groups in Fig. 5. As expected, $\zeta=-1$ networks are greatly influenced by the siting of generators. In fact, in centralized generator configurations the number of tie lines is minimal for this type of network. Even the initial double contingency, in some configurations, can disconnect entirely the supply nodes from the demand nodes. The other network topologies are not so sensitive, thanks to their $N$-1 compliant design, as even when all generators are clustered together, there is enough redundancy in the tie lines to prevent the complete power supply/demand disconnection (see Fig. 2 for clustering examples).

The layout of load and generators seem to affect the line outages $N_{\text {tot }}$ in all configurations, especially the $\zeta=0$ case. For $\zeta=-1$ the distributed generators slightly increase the quantity of line outages in the networks $N_{\text {tot }}$-an opposite trend with respect to $\zeta=\{0,1\}$. Overall, one notices that the role of $\zeta$, at low to medium levels that capture most realistic systems, is critical with respect to $K$ in determining the behavior of both power losses and line failures.

\subsection{Impact of re-dispatch policy}

In addition to the topological structure and electrical features of a power grid, the re-dispatch of power generation is one of the most frequently used control actions taken by operators to decrease operational costs and increase reliability (thus essential in the case of islanding [34]). When contingencies strike, re-dispatch is also applied as a mitigation action to avoid additional cascade failures; however, in some real fast-evolving cases, wrong re-dispatch can actually worsen the situation [38]. Fig. 6 shows the log-log plot of the tail distribution for 


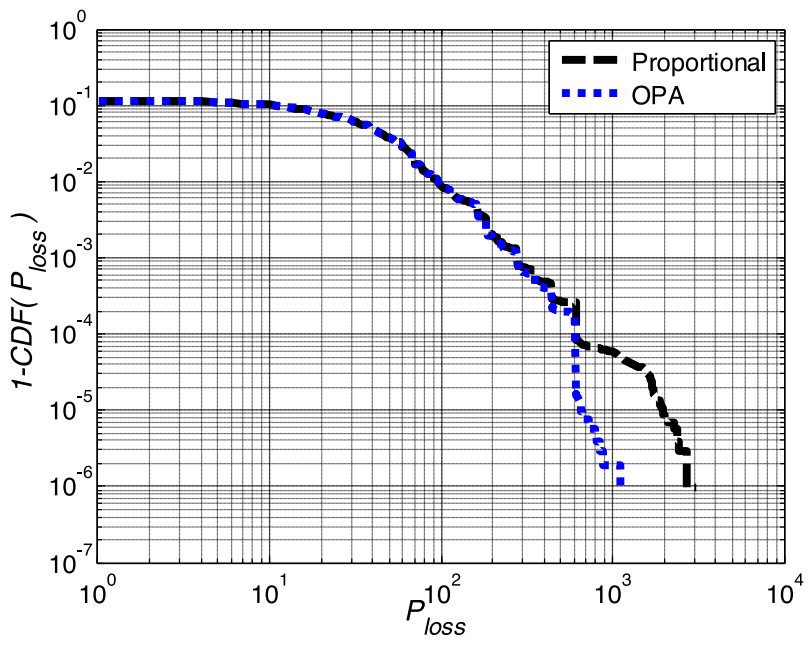

(a)

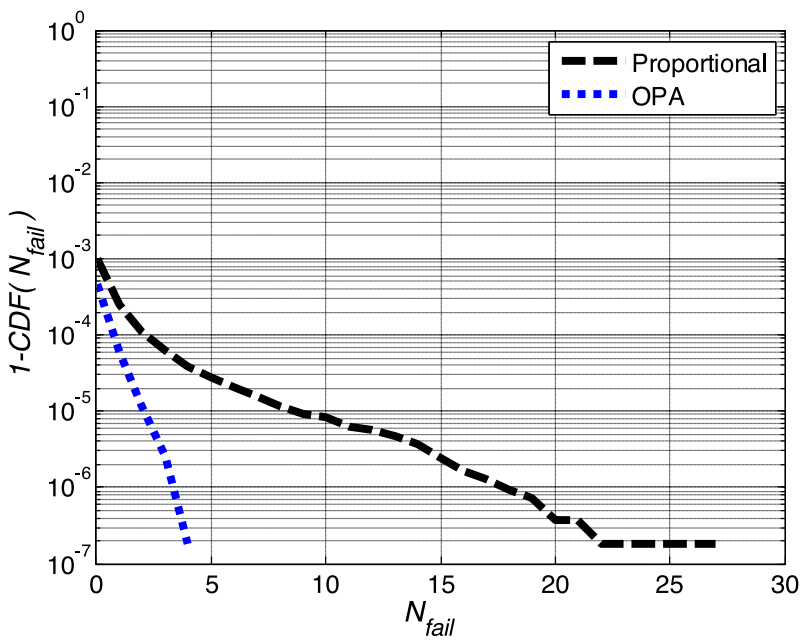

(b)

Fig. 6. Probabilistic response variables as a function of re-dispatch strategy: (a) log-log plot of the tail distributions of $P_{\text {loss }}$, and (b) semi-log plot for the tail distribution of $N_{\text {tot }}$.

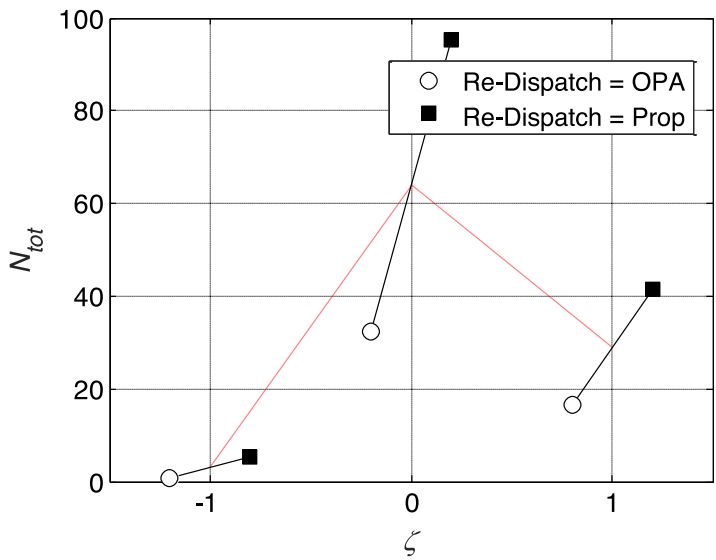

(a)

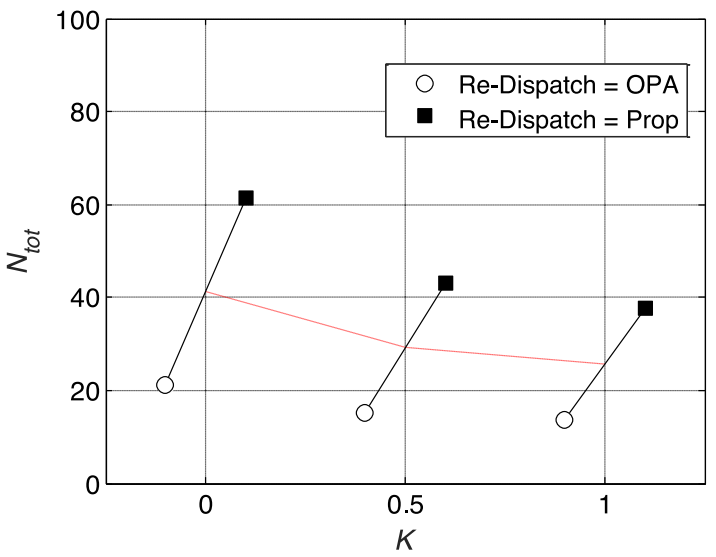

(b)

Fig. 7. Multivariate chart for identifying interactions between (a) re-dispatch and topology, and $(b)$ re-dispatch and generator siting $K$.

$P_{\text {loss }}$ and the semi-log plot of the tail distribution for $N_{\text {fail }}$, across cases which share the same re-dispatch procedure. In particular, we consider a proportional procedure and the OPA model. The impact of the more sophisticated OPA control strategy in preventing overloads is clear in Fig. 6(b). In fact, different from the proportional model which tries to accommodate the demand in a greedy way, the OPA model sacrifices power demand in order to prevent line failures whenever the linear program (12)-(18) is feasible, thus preventing cascading failures to spread. Regarding $P_{\text {loss }}$, OPA seems to succeed in reducing power losses in the most extreme scenarios, but does not have significant impact on small and medium power outages as evidenced in Fig. 6(a). This is because the OPA policy focuses on optimizing power losses under the constraint of no additional overloads (Eq. (16)), thus avoiding extended cascades which represents the extreme scenarios where large amounts of power are lost.

Fig. 7 shows the multivariate chart for $N_{\text {tot }}$, comparing the mean effect of the combinations of the re-dispatch factor with topology and generator position. The OPA re-dispatch successfully prevents line failures in all different topologies and also with respect to all generator configurations in $K$. Overall, the best improvement is obtained in the intermediate line redundancy level $\zeta=0$ which was identified as more vulnerable to line overloads (and the closest to redundancy levels in practice).
Meanwhile, $P_{\text {tot }}$ offers no discernible interaction pattern, mainly because as noted in Fig. 6, the main effect of the re-dispatch factor on $P_{t o t}$ is itself weak, influencing the outcomes of only rare scenarios.

\section{Insights for power grid design and operation}

This work shows that line redundancy and generator/load layout factors have a significant impact on the robustness of power grids to power loss and cascading line failures. From the line redundancy perspective, a minimum amount of redundant transmission lines is clearly necessary to avoid power interruptions every time a line fails. As shown in Fig. 3, if topologies are denser than the $\zeta=-1$ case (approaching the $\zeta=0$ layout), power losses decrease, although it is still possible to overload lines due to power flow re-distribution, and lead to widespread blackouts. At an extreme, if a considerable number of redundant lines are added (approaching the $\zeta=1$ layout), it becomes possible to do both: significantly decrease the overload frequency of the transmission lines and avoid large blackouts. In practice, however, building new transmission is too expensive to pursue a $\zeta=1$ power grid. Therefore, we identify a baseline combination of the topological factors of redundancy and generator layout which, while being attainable in practice, still achieve a good level of protection to cascading failures. To this end, we perform 


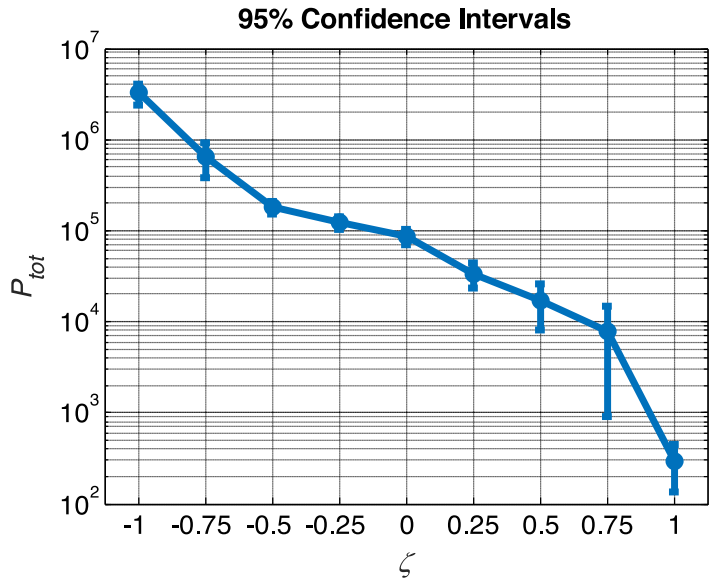

(a)

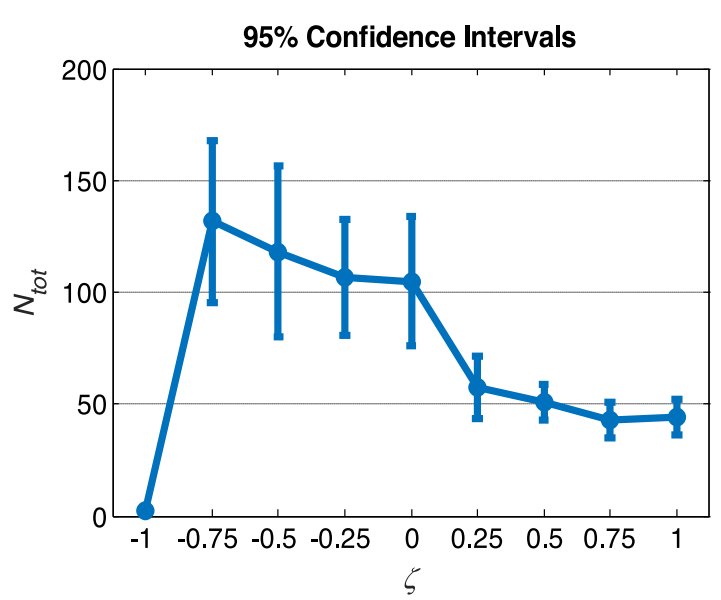

(b)

Fig. 8. Confidence intervals of the group mean of (a) $P_{t o t}$ and (b) $N_{\text {tot }}$ for different line redundancy values.

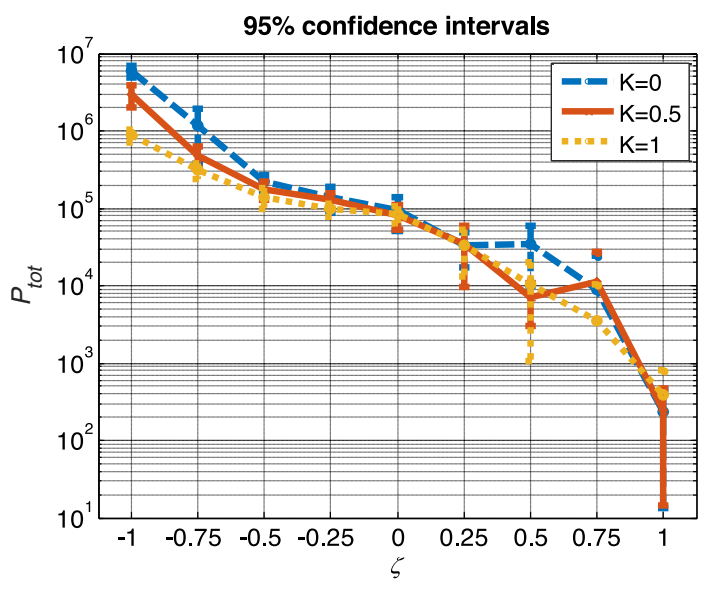

(a)

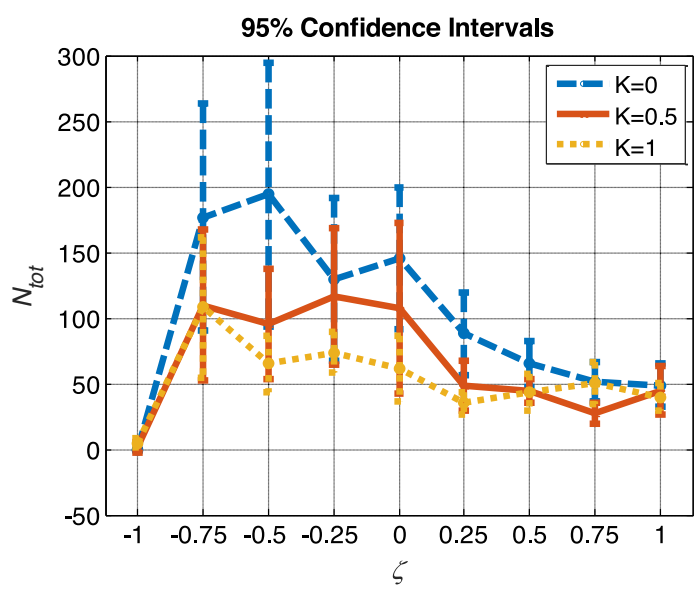

(b)

Fig. 9. Confidence intervals of the group means of (a) $P_{\text {tot }}$ and (b) $N_{\text {tot }}$ for different line redundancy values and generator layouts.

additional simulations with power networks with intermediate redundancy levels, in-between $\zeta=\{-1,0,1\}$. Since the design of the control strategy can be carried out independently from the choices of $\zeta$ and $K$, we perform simulations with the proportional re-dispatch only. In this way we obtain lower bounds on the power network robustness that can then be further improved by applying a more sophisticated control strategy, such as OPA (Figs. 6 and 7). Fig. 8(a) and (b) show the confidence intervals for the mean of $P_{\text {tot }}$ and $N_{\text {tot }}$ as we vary the redundancy level of the baseline case ( 0 on the $x$-axis) toward -1 or 1 with proportional re-dispatch.

Fig. 8(a) shows that adding lines always helps saving power losses as $P_{\text {tot }}$ is monotonically decreasing. The behavior of $N_{\text {tot }}$ is more complex and not monotonic as also evidenced in Fig. 3. Having only a few redundant lines above the $S T$ configuration leads to highly vulnerable topologies, while continuing to add more lines after the peak, slowly improves the situation (Fig. 8(b)). Also, after $25 \%$ of the lines which separate $G T$ from $G$ are added to the latter, significant improvement is achieved, but beyond that point improvement is marginal. Hence, building new transmission lines would not be the most economic and time efficient strategy to reduce the power grid risk of cascading failures for denser topologies, so measures relying on control strategies and/or distributed generators should also be included.

Considering the layout of loads and generators we show they affect mainly $N_{t o t}$. On average, more distributed configurations render less power loss and noticeably smaller cascading failures. The magnitude of the improvements depends on the redundancy level of the network. In particular, the lower the number of redundant lines in the grid beforee becoming trees, the more sensitive the response to generator layout. Fig. 9 shows the confidence intervals for the mean of $P_{t o t}$ and $N_{\text {tot }}$ as we vary the redundancy level of the baseline topology for the three different generators layouts. Looking at the confidence bands in Figs. 8(b) and 9(b), we notice how for $N_{\text {tot }}$ the intervals are wider for sparse topologies and clustered generators layouts.

These results suggest that dispersing power generating units across the power grid helps to avoid line overloads whenever the number of redundant lines is not sufficient. This type of intervention is happening ad-hoc via smart grid technologies and distributed generation (DG) today. Most likely, combinations of transmission line build up and DG, which require novel controls, offer a foreseeable solution to manage power losses and cascades in evolving power systems.

Regarding re-dispatch, we find it has a determinant role in avoiding the overload of additional transmission lines after initial contingencies materialize. Moreover, corrective re-dispatch actions interact in a synergistic way with the power grid topologies, bringing weaker configurations at approximately the same level of the stronger ones. In power grids vulnerable to overload, the optimized OPA model could generally avoid large scale cascades and associated blackouts, relative to simpler proportional strategies. 


\section{Summary and conclusions}

This study develops new strategies to evaluate cascading failure dynamics through a broad set of realistic power grid topologies operated with different power re-dispatch strategies. Cascading failures are sensitive to the initial power grid topology, supply/demand states, and the control actions applied while cascades evolve. Most existing work is specific to case study systems with set topology, and thus conclusions are typically not generalizable. The approach taken in this work is instead to explore key topological, electrical and control inputs across realistic power grids in order to find parameter settings to safeguard grids from cascading failures. The quantitative results drawn from this exploration are translated into high-level guidelines for reliability-based power grid design that are generalizable, since the global-to-local computational strategy employed here is probabilistic and accommodates an ensemble of topological and electrical system configurations.

Results show that for reducing the probability of large power losses, improvements at the structure/layout levels are necessary, including strategies that increase line redundancy, decentralize generators, or use combinations of them (at levels not too distant from realistic system configurations). Meanwhile, if major risks derive from too frequent overloads, it is better to inspect the control policies currently employed in the system and assess if it is necessary to improve or optimize them. In particular, power re-dispatch shows that line overload containment through optimized load shedding and power generation re-scheduling is effective in prevent the propagation of failures in all the power grid configurations considered.

Overall results show that the best practical solution to the problem of minimizing the probability of power losses and multiple line overloads is to have a topology slightly more redundant than the average level for realistic systems $0<\zeta<0.25$, coupled with distributed generators layout $K>0.50$. In addition to this topological solution, an optimized redispatch strategy would bring even greater benefits by curtailing the few cascading failures that still can breach a robust power network design. Also, increased line redundancy and generator decentralization levels reduce the variability of performance measures, such as the number of lines overloaded, furthering the manageability of complex power systems.

Future research includes extending the computational experiments presented in this work by considering AC power flow [34] and other forms of control actions such as those that modify the topology of the grid [35]. Moreover, instead of considering single or double contingencies, the initial failure events could be generated with hazard-consistent simulation approaches, so as to represent risk due to extreme events and quantify the associated uncertainty in the cascading dynamics of power grid systems. And risk analyses that exploit the hierarchical structure of networks also offer untapped opportunities for power systems [39].

\section{Notation list:}

\section{Factors}

$\begin{array}{ll}\zeta: & \text { line redundancy factor } \\ K: & \text { generator layout factor } \\ R: & \text { re-dispatch factor }\end{array}$

$\begin{array}{ll} & \text { Sets } \\ \mathbf{N}: & \text { set of power grid objects } \\ E: & \text { set of arcs of the graph representing the power grid topology } \\ V: & \text { set of vertices of the graph representing the power grid topol- } \\ & \text { ogy }\end{array}$

\begin{tabular}{ll}
\multicolumn{2}{c}{ Objects: } \\
$\mathcal{N}: \quad$ power grid object \\
$\mathcal{N}^{0}:$ & power grid object at initial state \\
$\mathcal{N}^{r}:$ & power grid configuration after the $r$ th line failure event
\end{tabular}

Power grid electrical parameters:

$Z$ : $\quad$ vector containing the impedances of the arcs
PD: vector containing the power demands for buses in the power grid

$P D_{i}: \quad$ power demand for bus $i$

PG: $\quad$ vector containing the power supplies for buses in the power grid

$P G_{i}: \quad$ power supply for bus $i$

PMAX: vector containing the power supplies limits for buses in the power grid

$P M A X_{i}$ : power supply limit for bus $i$

$C$ : $\quad$ vector containing the capacities for the arcs in the power grid

Power flow and capacity allocation parameters and variables:

$l$ : $\quad$ line of the power grid defined as an arc with impedance and capacity $(e, Z, C)$

$f_{l}$ : $\quad$ power flowing through line $l$

$f_{l}^{i}$ : $\quad$ power flowing through line $l$ when only line $i$ is failed

$f_{l}^{\max }$ : maximum power flowing through $l$ obtained from $N-1$ contigency analysis

$f^{r}: \quad$ power flow vector associated with $\mathcal{N}^{r}$

$\bar{C}_{i}$ : $\quad i$ th smaller capacity value that can be sampled from the capacity distribution

$C_{\zeta}^{\max }: \quad$ maximum capacity value allowed for a given value of line redundancy $\zeta$

\section{Re-dispatch parameters and variables:}

$\alpha_{i}$ : $\quad$ coefficient for proportional power generator adjustment for bus $i$ (proportional re-dispatch)

$\gamma_{i}: \quad$ constant coefficient for power generator adjustment for bus $i$ (proportional re-dispatch)

$\beta_{i}$ : $\quad$ coefficient for proportional power demand adjustment for bus $i$ (proportional re-dispatch)

$\Delta p g: \quad$ power generation adjustment vector (OPA re-dispatch)

$\Delta p d$ : $\quad$ power demand adjustment vector (OPA re-dispatch)

$\Delta p g_{i}: \quad$ power generation adjustment for bus $i$ (OPA re-dispatch)

$\Delta p d_{i}: \quad$ power demand adjustment for bus $i$ (OPA re-dispatch)

B: $\quad$ susceptance matrix

$\varepsilon_{l}: \quad$ noise in the capacity nominal value for line $l$

Response measures:

$P_{\text {loss }}^{\left(l_{i}, l_{j}\right)}$ : amount of power not supplied at the final state when $l_{i}, l_{j}$ are selected as initial failures

$N_{\text {fail }}^{\left(l_{i}, l_{j}\right)}:$ amount of lines failed at the final state when $l_{i}, l_{j}$ are selected as initial failures

$P_{\text {tot }}: \quad$ total power loss across $\mathrm{N}-2$ contingencies

$N_{\text {tot }}: \quad$ total cascading lines across $N-2$ contingencies

\section{Acknowledgments}

The authors would like to acknowledge the support of this research by the Politecnico di Milano, Rice University, the U.S. National Science Foundation (Grants CMMI-1436844 and CMMI-1541033 ), the U.S. Department of Defense through its Army Research Office (Grant W911NF- 13-1-0340 ), and the Data Analysis and Visualization Cyberinfrastructure funded by NSF under grant OCI-0959097.

\section{References}

[1] U.S. Department of Energy the SMART GRID. Communication 2010;99:48

[2] Bienstock D. Optimal control of cascading power grid failures. In: Proceedings of the IEEE conference on decision and control; 2011. p. 2166-73.

[3] Zio E, Sansavini G. Component criticality in failure cascade processes of network systems. Risk Anal 2011;31(8):1196-210.

[4] Mei S, Ni Y, Wang G, Wu S. A study of self-organized criticality of power system under cascading failures based on AC-OPF with voltage stability margin. IEEE Trans Power Syst 2008;23(4):1719-26.

[5] A Carreras B, Lynch VE, Dobson I, Newman DE. Critical points and transitions in an electric power transmission model for cascading failure blackouts. Chaos 2002;12(4):985-94.

[6] Haarla L, Pulkkinen U, Koskinen M, Jyrinsalo J. A method for analysing the reliability of a transmission grid. Reliab Eng Syst Saf 2008;93(February (2)):277-87. 
[7] Hernandez-Fajardo I, Dueñas-Osorio L. Probabilistic study of cascading failures in complex interdependent lifeline systems. Reliab Eng Syst Saf 2013;111(March):260-72.

[8] Motter AE, Lai YC. Cascade-based attacks on complex networks. Phys Rev E Stat Nonlinear Soft Matter Phys 2002;66(6):2-5.

[9] Crucitti P, Latora V, Marchiori M. Model for cascading failures in complex networks. Phys Rev E - Stat Nonlinear Soft Matter Phys 2004;69(4):045104 Pt 2.

[10] Pu C-L, Pei W-J, Michaelson A. Robustness analysis of network controllability. Phys- ica A 2012;391(18):4420-5.

[11] Buldyrev SV, Parshani R, Paul G, Stanley HE, Havlin S. Catastrophic cascade of fail- ures in interdependent networks. Nature 2010;464(April (7291)):1025.

[12] Wang Z, Scaglione A, Thomas RJ. Generating statistically correct random topologies for testing smart grid communication and control networks. IEEE Trans Smart Grid 2010;1(1):28-39.

[13] Pahwa S, Scoglio C, Scala A. Abruptness of cascade failures in power grids. Sci Rep 2014;4:3694.

[14] Kargarian A, et al. Toward distributed/decentralized DC optimal power flow imple- mentation in future electric power systems. IEEE Trans Smart Grid 2017(99) 1-1.

[15] Montgomery DC. Des Anal Exp 2012;2.

[16] Hines P, Cotilla-Sanchez E, Blumsack S. Do topological models provide good infor- mation about electricity infrastructure vulnerability? Chaos 2010;20(3):1-5.

[17] Pagani GA, Aiello M. The Power Grid as a complex network: A survey. Physica A 2013;392(11):2688-700.

[18] Fang Y, Sansavini G. Optimizing power system investments and resilience against attacks. Reliab Eng Syst Saf 2017;159(March (Supplement C)):161-73

[19] Bier VM, Gratz ER, Haphuriwat NJ, Magua W, Wierzbicki KR. Methodology for iden- tifying near-optimal interdiction strategies for a power transmission system. Reliab Eng Syst Saf 2007;92(September (9)):1155-61.

[20] Dobson I, Carreras BA, Lynch VE, Newman DE. An initial model for complex dynam- ics in electric power system blackouts. In: Proceedings of the 34th annual hawaii international conference on system sciences; 2001. p. 710-18.

[21] Watts DJ, Watts DJ, Strogatz SH, Strogatz SH. Collective dynamics of 'smallworld' networks. Nature 1998;393(6684):440-2.

[22] Albert R, Barabasi AL. Statistical mechanics of complex networks. Rev Modern Phys 2002;74(1):47-97.

[23] Cotilla-Sanchez E, Hines PDH, Barrows C, Blumsack S. Comparing the topological and electrical structure of the North American electric power infrastructure. IEEE Syst J 2012;6(4):616-26.

[24] Purchala K, Meeus L, Van Dommelen D, Belmans R. Usefulness of DC power flow for active power flow analysis. Power Eng Soc General Meeting 2005:454-9.
[25] Hu J, Sankar L, Mir DJ. Cluster-And-connect: A more realistic model for the elec- tric power network topology. In: 2015 IEEE international conference on smart grid communications, SmartGridComm 2015; 2016. p. 85-90.

[26] Gegner KM, Birchfield AB, Xu T, Shetye KS, Overbye TJ. A methodology for the cre- ation of geographically realistic synthetic power flow models. In: 2016 IEEE power and energy conference at illinois (PECI); 2016. p. 1-6.

[27] "Power system test archive, UWEE." [Online]. Available: https://www.ee.washington.edu/research/pstca/.

[28] Zimmerman RD, Murillo Sánchez CE, Thomas RJ. MATPOWER: Steady-state opera- tions, planning, and analysis tools for power systems research and education. Power Syst IEEE Trans 2011;26(1):12-19.

[29] Hespanha P. An efficient MATLAB algorithm for graph partitioning technical report graph partitioning. October 2004:1-8.

[30] Li J, Dueñas-Osorio L, Chen C, Berryhill B, Yazdani A. Characterizing the topologi- cal and controllability features of U.S. power transmission networks. Physica A Jul. 2016;453:84-98.

[31] Cetinay H, Kuipers FA, Mieghem PV. A topological investigation of power flow. IEEE Syst J 2017(99):1-9.

[32] Zio E, Piccinelli R. Randomized flow model and centrality measure for electrical power transmission network analysis. Reliab Eng Syst Saf 2010;95(4):379-85.

[33] Dueñas-Osorio L, Vemuru SM. Cascading failures in complex infrastructure systems. Struct Saf 2009;31(2):157-67.

[34] Allen J Wood BFW. Power generation operation and control. 2nd Ed. WILEY-INTERSCIENCE; 1996.

[35] Zhang C, Wang J. Optimal transmission switching considering probabilistic reliabil- ity. IEEE Trans Power Syst 2014;29(2):974-5.

[36] Bienstock D, Mattia S. Using mixed-integer programming to solve power grid black- out problems. Discrete Optim 2007;4(1):115-41.

[37] Ouyang M HL, Mao Z, Yu M, Qi F. A methodological approach to analyze vulnera- bility of interdependent infrastructure. Simul Modell Pract Theory 2009;17(5):12.

[38] Andersson G, et al. Causes of the 2003 major grid blackouts in North America Europe, and recommended means to improve system dynamic performance. IEEE Trans Power Syst 2005;20(4):1922-8.

[39] Gómez C, Sanchez-Silva M, Dueñas-Osorio L, Rosowsky D. Hierarchical infrastruc- ture network representation methods for risk-based decision-making. Structure and Infrastructure Engineering 2013;9(3):260-74. 